\title{
Design and Implementation of Dynamic Deduction System for Emergency Drills of Waterlogging Disaster
}

\author{
Peng Chen ${ }^{1}$, Jiquan Zhang ${ }^{2}$, Yingyue Sun ${ }^{1}, J_{i a n h u a ~ B a o}{ }^{1}$, Shuo Zhang ${ }^{1}$, Hongyang Zhao ${ }^{1}$ \\ ${ }^{1}$ College of Tourism and Geographical Science, Jilin Normal University, Siping 136000, China \\ ${ }^{2}$ School of Environment, Northeast Normal University, Changchun 130117, China
}

\section{内涝灾害应急演练方案动态推演系统设计与实现}

\author{
陈鹏 ${ }^{1}$, 张继权 ${ }^{2}$, 孙港悦 ${ }^{1}$, 鲍建华 ${ }^{1}$, 张硕 ${ }^{1}$, 赵洪阳 ${ }^{1}$ \\ ${ }^{1}$ 吉林师范大学旅游与地理科学学院, 吉林 四平, 136000 \\ ${ }^{2}$ 东北师范大学环境学院, 吉林 长春 130117 , 中国
}

\begin{abstract}
In recent years, urban waterlogging is frequent, resulting in greater and greater losses. The disaster emergency drill is an important measure to reduce the losses caused by disasters, but the traditional emergency drill is not only time-consuming, but also wastes a lot of manpower, money and material resources. In view of this, the waterlogging disaster emergency rehearsal is the research object. Based on the technology of military chess, programming technology and GIS technology, combined with the content of emergency drill, the dynamic inference system of waterlogging disaster emergency rehearsal program is constructed. The system can realize the dynamic rehearsal of the emergency rehearsal process of waterlogging disaster and the evaluation of the result. The results can provide a platform for emergency departments to promote high risk events in low risk situations, and also improve command ability and awareness of overall situation.
\end{abstract}

Keywords: waterlogging; emergency rescue; war game; emergency drills
摘要

近些年城市内涝灾害频发, 造成的损失 越来越大。灾害应急演练则是减少灾害造成 损失重要措施, 但传统的应急演练不仅耗时 长, 还浪费大量人力、财力、物力。鉴于此, 以内涝灾害应急演练为研究对象, 以兵棋技 术、编程技术及 GIS 技术为基础, 结合应急 演练内容综合构建内涝灾害应急演练方案 动态推演系统, 该系统可实现内涝灾害应急 演练过程动态推演及其结果评估。研究结果 可为应急部门在低风险的情况下推演高风 险事件提供平台, 同时还可提高指挥人员的 指挥能力及大局观意识。

关键词: 内涝灾害; 应急救援; 兵棋; 应急 演练

近年来, 随着城市快速发展, 城市内涝 灾害频发, 给城市基础设施、财产及人身安 全造成严重威胁, 同时也严重阻碍了城市健 康发展。因此, 应急演练工作已经引起了各 级政府部门普遍重视。构建一套动态的、可 视化的内涝灾害应急演练动态推演系统对 快速检验应急预案合理性具有重要意义。

目前, 国内外已经广泛开展应急演练, 应急演练类型分为桌面型演练与实战演练 [1], 其中实战演练强调的是真实性, 会调用 
大量的人力、物力参与应急演练, 同时还需 要特定的场所, 一般应急演练过程持续时间 较长, 短则几小时, 长则达几十个小时, 因 此, 实战演练投入的成本较高; 而桌面演练 则可以充分利用流程图、沙盘、计算机模拟、 视频会议等技术及工具[2-4]。桌面推演除了 上述内容外, 还需要针对灾害或突发事件的 应急处置流程, 分别设置应急单位、应急决 策者及多方协同部门等角色 [5-6]。桌面演练 之前需要进行人员培训及命令下发, 推演过 程中与实战推演不同的是, 并不需要调用大 量人力、物力及财力, 具有占地小、成本少 的特点。但桌面推演缺乏动态性及无法设置 与处置突发事件等缺点。国外针对灾害管理 或模拟系统应用较为成熟的是美国 ECT 公 司开发的 ADMS 灾害管理模拟系统, 该系统 是一套服务于桌面演练的系统平台, 该平台 能够实现多种灾害的仿真模拟与应急演练, 目前该套系统已经在北美、亚洲及欧洲 30 多个国家的应急部门广泛应用[7-9]; 我国针 对灾害应急演练兵棋系统构建研究发展较 晚[10], 台湾在 90 年代初期陆续开展了灾害 兵棋推演系统研制方法方面开展了相关研 究, 在推演体系整体规划与系统开发上采用 了与地理信息系统技术相结合, 初步实现了 灾害兵棋推演系统。亦有学者将内涝灾害兵 棋推演相关研究主要应用在内涝灾害的应 急救援方面、应急管理及动态推演结果评估 上, 而并未见内涝灾害应急演练方案动态推 演及系统集成中[11-14]。

本文从内涝灾害发生、发展过程及应急 演练的特点出发, 集成计算机技术、网络技 术、通讯技术及 GIS 等多种先进技术, 围绕 平台的功能分析与结构设计, 综合构建内涝 灾害应急演练方案动态推演系统平台。内涝 灾害应急演练方案动态推演系统平台的构 建, 将弥补该方面的不足, 为城市内涝灾害 应急推演及其检验应急预案的合理性提供 动态的、可视化及智能化的推演系统平台。

\section{1. 系统架构}

\section{1 功能需求}

内涝灾害应急演练方案动态推演系统 是以严格式兵棋理论为指导, 依托 $\mathrm{C} / \mathrm{S}$ 体系
架构, 为满足内涝灾害应急演练方案动态推 演指挥训练要求, 应考虑对已有仿真训练资 源的复用, 本系统需具备以下功能:

(1) 可靠的演练方案动态推演建模方 法

计算机兵棋推演系统应能对战场空间 出现的各种要素、环境可以进行抽象和描 述, 构建全面一致的应急演练动方案动态推 演模型，同时需兼顾推演模型的通用性与可 扩展性, 以此来应对各种推演过程中遇到的 突发事件, 以及不同作战想定下的训练需 要。

（2）友好的人机交互界面

友好的人机交互界面可避免使用者可 能出现的误操作, 还可为用户提供方便、美 观、可视化的界面。本系统实现的是内涝灾 害应急演练方案兵棋推演, 因此, 系统界面 除了符合应急管理人员常用的习惯, 还需要 满足类似实装指挥控制系统的人机交互环 境各种虚拟作战实体进行指挥控制。

（3）高效合理的推演方法

内涝灾害应急演练方案兵棋推演系统 应能合理表现各种用户所需推演内容, 模拟 作战环境, 且及时、准确的解决各种突发事 件, 较为真实的用虚拟仿真的方法真实的体 现参训人员的指挥意图以及公平合理的作 战结果评判。

\section{2 系统结构}

一个完整的作战系统包括导训调理、指 挥系统、作战演练、辅助系统、数据准备及 运行支撑环境 6 个子系统。但这是军事上的 系统结构, 不符合本研究实际内容 [15]。因 此, 将内涝灾害应急演练过程与兵棋推演相 结合, 设定 4 个子系统, 包括指挥系统、辅 助评估系统、应急演练作战系统及运行支撑 环境。其中, 指挥系统是指挥整个应急演练 过程中包括人员如何调动与资源如何调动, 以及为参见演练人员提供图形化操作界面 与控制指挥命令; 而辅助评估系统是对推演 结果评估, 亦是完成应急演练方案篮选的重 要组成部分; 应急演练作战系统是处理指挥 子系统下达的控制命令和指挥命令, 并负责 推演计算。主要从内涝灾害的准备阶段、实 
施阶段及评估阶段是三个方面出发, 按照应 急演练流程设计子系统功能; 支撑环境子系 统是系统运行过程中维持系统正常运转的 重要系统, 包括对象类描述棋子状态, 使用 交互类描述作战指挥命令。

\section{2. 建模方法}

评判一个系统的好坏, 其建模方法的选 择非常重要。本研究采用的建模方法主要是 依据兵棋理论及方法以及利用面向对象的 设计思想, 综合构建内涝灾害应急演练方案 动态推演系统。

\section{1 作战实体建模}

兵棋推演中的作战实体为棋子, 每个兵 棋棋子代表一个作战单位, 并标有攻击值、 防御值、机动点值、棋子类型、主要装备、 作战代号及棋子编号等主要信息。对于内涝 灾害应急演练过程中, 设定我方棋子 (应急 单位) 属性为救援能力值、机动点值、棋子 类型、防御值、棋子编号; 而敌方棋子为内 涝灾害, 因此敌方棋子属性较为简单, 包括 范围变化值、水深、流速。由于敌方棋子无 法移动, 就不存在机动点值与类型。具体设 定见表 1 .

表 1 . 棋子属性设定

\begin{tabular}{|c|c|c|}
\hline $\begin{array}{l}\text { 类 } \\
\text { 型 }\end{array}$ & $\begin{array}{l}\text { 参数 } \\
\text { 名称 }\end{array}$ & 参数描述 \\
\hline $\begin{array}{l}\text { 属 } \\
\text { 性 }\end{array}$ & $\begin{array}{l}\text { 单位名称 } \\
\text { 编号 } \\
\text { 类型 }\end{array}$ & $\begin{array}{l}\text { 作战单位名称 } \\
\text { 作战单位编号 } \\
\text { 棋子类型, 如救护单位、运输 } \\
\text { 单位、清除积水单位等。 }\end{array}$ \\
\hline $\begin{array}{l}\text { 能 } \\
\text { 力 }\end{array}$ & $\begin{array}{l}\text { 救援能力 } \\
\text { 防御能力 } \\
\text { 机动能力 } \\
\text { 通信能力 }\end{array}$ & $\begin{array}{l}\text { 清除积水能力、救助灾民能力 } \\
\text { 等。 } \\
\text { 指应急单位承受灾害影响能 } \\
\text { 力。 } \\
\text { 应急单位机动点数, 如移动值。 } \\
\text { 在应急演练过程中, 指挥中心 } \\
\text { 下达命令后, 信息传输的及时 } \\
\text { 性。 }\end{array}$ \\
\hline $\begin{array}{l}\text { 状 } \\
\text { 态 }\end{array}$ & $\begin{array}{l}\text { 棋子状态 } \\
\text { 后勤保障 } \\
\text { 状态 }\end{array}$ & $\begin{array}{l}\text { 棋子自身的状态, 如死亡、受 } \\
\text { 伤。 } \\
\text { 后勤保障能力及水平 }\end{array}$ \\
\hline
\end{tabular}

\section{2 作战活动建模}

军事上的作战推演是双方指挥人员控 制, 发布一系列指挥控制命令。而本系统中 的敌方是内涝灾害, 因此并不能设置敌方指 挥命令, 仅能设置内涝灾害随时间变化代替 敌方指挥。我方指挥控制主要是针对应急单 位, 即我方棋子的位置、机动值及救援等则 是指挥命令。敌

\section{3 作战环境建模}

作战环境是兵棋推演的重要场所, 本系 统采用矢量地图, 并辅以六角格作为兵棋地 图。

其中矢量地图是利用 ARCGIS 技术将研 究区遥感影像进行解译, 获取而来, 因矢量 数据本身就具备属性这一特点, 所以可以很 好的仿真实际地理环境。六角格是棋子定位 和走棋的基本单元, 其绘制过程亦是采用 ARCGIS 技术, 并将矢量地图与六角格进行 拓扑关系, 使得六角格具备矢量地图基本信 息, 为棋子行动提供基础数据依据。

\section{3. 子系统设计}

本系统的推演原则是以回合制进行推 演, 在一个回合内不在依据作战原则进行详 细划分, 同时走棋亦不受走棋轮次限制。但 为了更好的表达人与自然灾害的“博弯”过 程, 以内涝灾害演变时间为主线, 将时间加 入控制指挥命令, 同时记录指令下达时间、 行动持续时间与回合作战完成时间。以应急 车辆为例, 因研究区高程差较少, 地势较为 平坦, 车辆移动相对不受过多限制, 设定车 辆移动速度 $60 \mathrm{~km} / \mathrm{h}$, 地图 1 格表明实地 $6 \mathrm{~km}$, 则 $1 \mathrm{~h}$ 应急车辆将移动 10 格, 车辆每格移动 需消耗 $10 \mathrm{~min}$, 这亦成为回合时间, 因此, 本系统将以 $10 \mathrm{~min}$ 来衡量 1 回合时间内基本 应急单位可完成的最基本状态, 作战系统便 可进行有意义的态势生成计算, 其他子系统 也将遵守此原则。

\section{1 指挥控制系统}

指挥控制系统是以内涝灾害演变时间 线, 按照每 $10 \mathrm{~min}$ 为步长, 一一发布相应指 挥控制命令, 该系统将由指挥中心登录, 并 
由指挥者依据内涝灾害发展态势进行命令 下达。该子系统包括模块功能如下:

(1) 人机交互界面。负责显示我方应 急单位实时状态及情报, 为指挥者提供指挥 命令下达依据;

(2) 数据管理。本系统中使用的数据 包括基础地理信息数据（属性数据、空间数 据)、降雨数据、社会经济数据。不同类型 数据分别存储在后台的属性数据库与空间 数据库中, 以便其他子系统进行调用;

（3）情报管理。负责管理各单位上报 的应急及伤亡情报, 并进行分类处理, 为后 续的推演结果评估提供依据;

（4）指挥命令下达管理。系统内部设 定一个定时发送线程，定时申请作战时间推 进，同时发送本周期内产生的指挥控制命 令。

\section{2 应急演练作战系统}

应急演练作战系统是整个推演系统的 核心，包括基础服务层、业务层、推演管理 层三个层次。为配合三个层次设定 5 个基本 功能模块, 具体如下:

（1）想定文件管理。想定文件如一部 电影的剧本, 将整个推演过程中的敌我方行 动过程、具体指挥过程及推演过程都采用想 定编写成具体内容, 并进行统一管理;

（2）业务管理。负责管理棋子属性数 据与兵棋地图数据;

（3）作战实体管理。系统中所有作战 实体状态数据都在本功能模块内进行统一 管理, 为后续推演提供数据支持;

(4) 命令处理。负责将指挥系统发布 的指令传送到其他子系统, 并进行指挥命令 管理;

（5）推演线程管理。包括推演过程计 算管理、内部维护及时间计算器等作战推演 辅助管理;

（6）推演算法管理。为实现内涝灾害 应急演练方案动态推演, 将描述推演过程的 算法、流程及各种裁决过程使用方法等进行 统一管理。

\section{3 辅助评估系统}

辅助评估系统是对推演结果进行评估, 是实现内涝灾害应急演练方案好坏的评判 依据。本子系统主要对各系统的推演过程及 辅助过程进行评判, 每个评判过程均报送指 挥中心, 由指挥中心统一管理。

\section{4. 系统实现}

以内涝灾害应急演练过程为依据, 结合 实际应急演练方案, 确定内涝灾害应急演练 推演过程分为四个过程, 即准备阶段、推演 阶段、反馈阶段及评估阶段。本系统平台实 现是以 JavaScript、ArcSDE 及数据库技术综 合构建，具体系统框架如图 1 所示。

\section{1 准备阶段}

\section{1 .1 数据准备}

本系统包括数据较多，其中属性包括社 会、经济、气象、排水管网以及应急单位信 息等, 而空间信息包括研究区矢量地图数 据、六角格数据、及下垫面基础数据。大量 信息数据管理采用属性数据库及空间数据 库共同完成管理，以备其他子系统调用。

\section{1 .2 文件准备}

目前本系统中的描述文件里有 11 个 csv 文件与 1 个 js 文件 (见图 2)。其中 11 个 csv 文件分别是实现了包括走棋、行动及地图基 础地理信息。而 js 文件主要用来起到调用这 些文件用以实现各文件的指令。具体文件包 括如下:

(1) AI.csv 描述棋子行为方式;

(2) block.csv 描述遮罩地图方式，描 述启用地图的哪一部分;

（3）capture.csv 描述地图上各六角格 所属关系, 比如棋子位置;

(4) color.csv 描述算子颜色的样式;

(5) CRT.csv 描述战斗结果表;

（6）label.csv 描述地图上一些六角格 的特殊名称;

(7) player.csv 描述用户级的一些数 据, 比如移动指令;

（8） script.js 事件触发器;

（9） setting.js 一些指导系统采用什么 模式, 怎么处理一些具体情况的描述文件; 


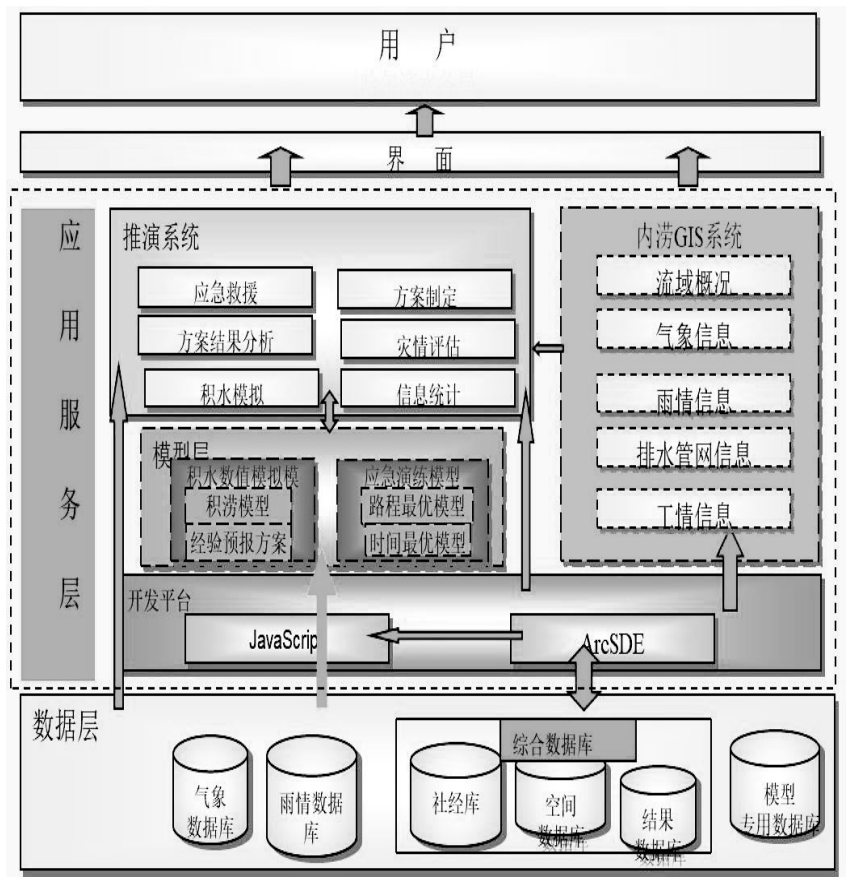

图 1. 系统框架

(10) terrain.csv 描述各六角格地形;

(11) unit.csv 描述单位信息与布置;

(12) VP.csv 描述各六角格内信息的变 化。

㞋称

a.j. Al.csv

abd block.csv

a. capture.csv

a. color.csv

ab. CRT.CSV

a. label.csv

a. player.csw

술ㅇ script.js

$\Rightarrow$ setting.csw

a. terrain.csv

a.j unit.csv

a.d VP.csV

\section{图 2. 系统后台文件}

\subsection{3 模型准备}

本系统中关键模型包括三个, 即内涝灾 害演变模型、推演模型及评估模型, 三个模 型在作者主持项目中已经分别构建完成, 已 经将三个模型嵌入到本系统中。其中内涝灾 害演变模型主要实现了研究区内容道路积 水变化过程, 用以设定敌方行动及其规则;
推演模型实现了内涝灾害应急演练的动态 推演, 模拟了应急部门对内涝灾害应急演练 的全过程; 评估模型主要实现了每个回合及 最终推演结果进行评估, 为推演者提供直接 可视化的结果, 并得到一次内涝灾害应急演 练的结果。结果以百分制体现, 让推演者清 晰的了解某个应急演练方案制定的优缺点, 为后续的整改提供决策依据。

\section{2 推演阶段}

以想定背景为基础推演背景, 以基础数 据、文件及模型为依据, 采用 $\mathrm{B} / \mathrm{S}$ 结构, 即 Browser/Server(浏览器/服务器)结构实现本 系统。本系统推演过程实现了内涝灾害应急 演练的前期准备、演练实施及评估三个阶 段。具体实现过程为用户通过指定权限用户 登录网址, 分别扮演不同身份推演者, 包括 指挥者、裁决者、推演者及应急单位等; 登 录完成后, 按照系统提示信息开始设定推演 情景、推演流程、推演棋子属性及内涝灾害 演变情景; 开始推演时, 由指挥者发布开始 


\section{a dinumbs}

Advances in Economics, Business and Management Research, volume 66

推演指令及下达指挥命令, 各部门将按照指 令进行推演; 裁决者将通过信息反馈子系统 将反馈信息进行裁决, 并填写对战报告; 最 后, 所有推演信息送达至评估子系统, 依据 反馈子系统填写的表单, 评估子系统会进行 打分, 以确定本系内涝灾害应急演练最终得 分, 具体系统推演界面见图 3。

\section{5. 结论与讨论}

\section{1 结论}

随着计算机兵棋推演技术在军事上的 广泛应用, 对锻炼指挥人员的指挥能力和大 局意识起到了重要作用。将这一技术引入到 内涝灾害应急演练中, 正是体现了此方面的

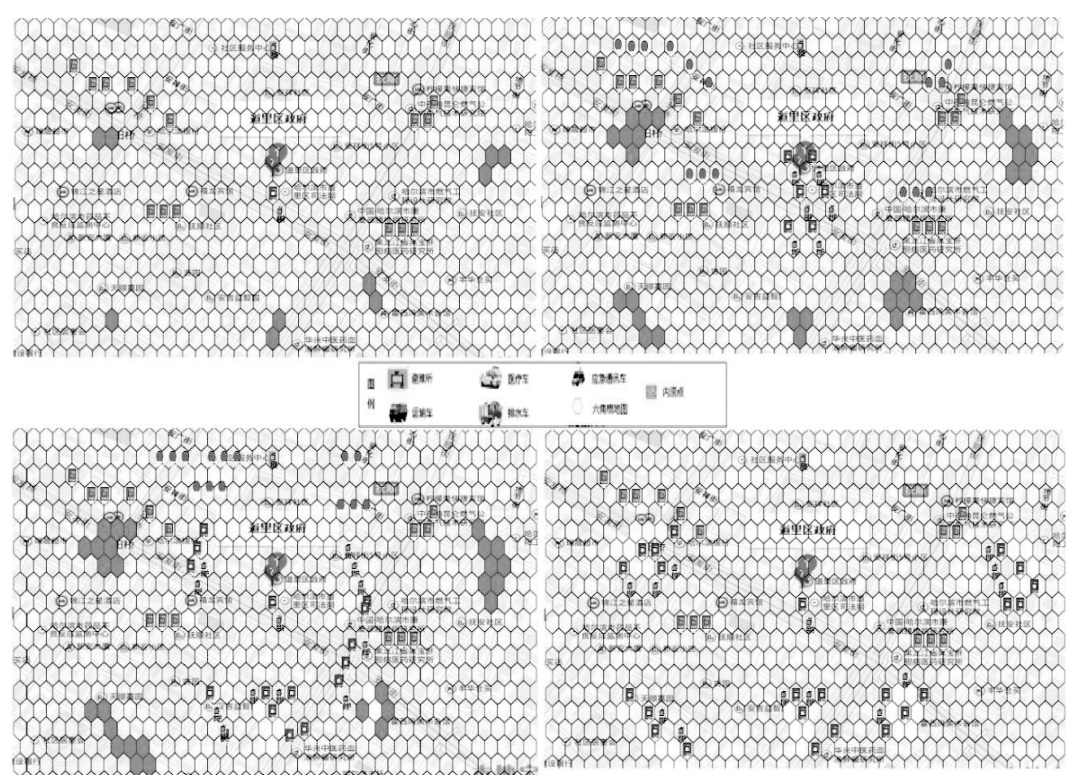

图 3. 内涝灾害应急预案动态推演系统截图

\section{3 反馈与评估阶段}

反馈子系统是辅助评估子系统完成对 推演结果的评价, 该子系统与其他两个子系 统亦有关联, 主要是从推演子系统中获取内 涝灾害演练过程中的结果信息, 实现将结果 信息进行收集与传递功能。评估子系统将获 取到的内涝灾害应急演练过程中的相关信 息传递给评估模型, 便可实现对一次内涝灾 害应急演练过程的评估。评估结果按百分制 进行赋分, 评估结果按照优 (90-100 分)、 良 (80-90 分)、中 (70-80 分)、差 (60-70 分) 四个等级进行划分。评估子结果可为城 市内涝灾害应急部门有针对性的调整应急 预案内容, 为更好的进行应急演练提供决策 依据。
优点。本文从系统架构、建模方法、子系统 设计及系统实现四个方面构建了内涝灾害 应急演练动态推演系统。该系统是一套复杂 系统, 是由政府部门为主导, 大量相关人员 参与的系统, 可检验内涝灾害应急演练的过 程的优缺点, 为相关应急决策者及应急单位 提供可视化、智能化与自动化的推演系统。

\section{2 讨论}

本系统虽构建与实现了内涝灾害应急 演练过程动态推演与评估, 系统运行较为正 常, 但系统中实测数据不足, 内涝灾害模拟 精度存在一定误差。另一方面, 由于系统中 的推演命令是参考相关研究与咨询专家综 合设计得到, 因此, 主观影响因素较多, 需 要今后研究中进一步通过实证数据加以验 证, 使得命令设定更为符合实际。 


\section{致谢}

本研究得到了国家社会科学基金项目 (41501557)的资助。

\section{参考文献}

[1] 姜传胜,邓云峰,贾海江,等.突发事件应 急演练的理论思辨与实践探索.中国安 全科学学报,2011,21(6):153-159.

[2] Ralph Renger, Jessica Wakelee,Jillian Bradshaw. The hybrid exercise: Transitioning from discussion-based to operations-based exercises, Journal of Emergency Management, 2009, 7(4) : 51-56.

[3] U.S.Department of Homeland Security, Homeland Security Exercise and Evaluation Program ( HSEEP) .2013.

[4] George D. Haddow, Jane A .Bullock, Damon P. Coppola. Introduction to Emergency Management. Fourth Edition. USA:ELSEVIE, 2011,12-20.

[5] 李群,代德军.突发事件应急演练评估方 法、技术及系统研究.中国安全生产科学 技术,2016,12(07):49-54.

[6] 黄思成, 林子博, 林欣琪, 李玲, 王晓霖, 陈 思东.突发公共卫生事件双盲应急综合 演练评估体系构建. 职业卫生与应急救 援,2017,35(06):575-578.

[7] 杨德屏,郭红玉,熊治,等.基于全生命周 期的企业应急演练效果评估体系研究. 工业技术创新,2016,03(04):702-706.

[8] 王文俊,张迎军,金雪松,等.基于 GIS 的机 场应急救援演练系统开发与应用研究. 中国安全生产科学技术,2017,13(03):16 9-173.

[9] 马叶钦,王红卫,祁超. 基于计算机仿真的 桌面演练研究. 管理评论,2016,28(08):18 6-192.

[10] Lakshay, Amit Agarwal, Nomesh B. Bolia. Route Guidance Map for Emergency Evacuation. Journal of Risk Analysis and Crisis Response, 6(3): 135-144.
[11] 陈鹏, 张继权,孙滢悦, 等.城市内涝灾害 居民应急避难兵棋推演理论与方法. 人 民长江, 2016, 47(14):7-11.

[12] Peng Chen, Jiquan Zhang,Lifeng Zhang and Yingyue Sun. Wargame Simulation Theory and Evaluation Method for Emergency Evacuation of Residents from Urban Waterlogging Disaster Area, International Journal of Environmental Research and Public Health, 2016, 13(12):2-19.

[13] 陈鹏,孙漟悦,刘晓静,邹桃红.城市内涝 灾害社会影响评价研究.吉林师范大学 学报(自然科学版),2018,39(01):135-140.

[14] 周柏贾,贾群林.地震应急演练虚拟仿真 应用技术.自然灾害学报,2011,20(05):59 $-64$.

[15] Peng Chen, Jiquan Zhang, Yingyue Sun, Xiaojing Liu. Wargame Mapping and Implementation for Emergency Evacuation of Residents in Urban Waterlogging Disaster. Journal of Risk Analysis and Crisis Response, 2018, 8(1):43-51. 